\title{
Gefahr einer Mangelernährung älterer Patient_innen im Akutspital
}

\author{
Implikationen für die Pflegepraxis
}

\author{
Silvia Brunner ${ }^{1,2}$ (D), Hanna Mayer ${ }^{1}$, Michael Dietrich²,3, Matthias Breidert ${ }^{2,4}$, Karin Blum², \\ Maria Müller-Staub ${ }^{5}$ \\ ${ }^{1}$ Institut für Pflegewissenschaft, Universität Wien \\ ${ }^{2}$ Stadtspital Zürich \\ ${ }^{3}$ Medizinische Fakultät der Universität Zürich \\ ${ }^{4}$ Technische Universität München \\ ${ }^{5}$ Hanze University Groningen
}

Zusammenfassung: Hintergrund: Die Gefahr einer Mangelernährung ist bei hohem Alter und akuter Erkrankung erhöht und deren Erfassung sowie bedarfsgerechte Unterstützung gehören zum Verantwortungsbereich der Pflege. Fragestellung/Ziel: Mit der folgenden Forschungsfrage sollen Ernährungszustand sowie mögliche Zusammenhänge zu Pflegediagnosen und weiteren Patient_innen-Merkmalen von Personen (80-jährig und älter) analysiert werden: Welche Muster im Sinne von Zusammenhängen lassen sich in Bezug auf Kalorien- und Proteinbedarfsdeckungsgrade und weiteren Patient_innen-Merkmalen erkennen? Methoden: Explorative Querschnittsstudie mit Clusteranalyse auf Grundlage von Essprotokollen und Pflegedokumentationen. In die Gelegenheitsstichprobe eingeschlossen wurden Patient_innen der Behandlungsbereiche Chirurgie, inneren Medizin und universitäre Akutgeriatrie. Ergebnisse: Aus Daten von 135 Patient_innen wurden vier Gruppen gebildet (Proteinbedarfsdeckungsgrad): Gut-Ernährte (116\%), Genügend-Ernährte (77\%), Ungenügend-Ernährte (59\%) und SchlechtErnährte (40\%). Ein signifikanter Zusammenhang von Kalorien-und Proteinbedarfsdeckungsgrad und Behandlungsbereich konnte gezeigt werden. Schlussfolgerungen: Protein- und Kalorienbedarfsdeckungsgrade hängen mit dem Behandlungsbereich und folglich mit dessen Teamkultur und Behandlungskonzept zusammen. Aufgrund der Eigenschaften der Gut-Ernährten kann auf eine positive Wirkung der interprofessionellen Zusammenarbeit und der systematischen Erfassung der Gefahr einer Mangelernährung, wie es im Bereich Akutgeriatrie umgesetzt wird, geschlossen werden.

Schlüsselwörter: Ältere Patient_innen, Pflegediagnosen, Gefahr der Mangelernährung, Protein- und Kalorienbedarfsdeckungsgrad, Akutspital

\section{Risk of malnutrition in elderly patients in acute hospitals - implications for nursing practice}

\begin{abstract}
Background: The risk of malnutrition is increased in advanced age and acute illness, and its assessment and needsbased support are part of the responsibility of nursing. Research question/objective: The following research question aims to analyse the nutritional status and possible correlations with nursing diagnoses and other patient characteristics from persons who are 80 years old and older: Which patterns in the sense of clusters can be identified concerning calorie and protein requirements and other patient characteristics? Methods: Explorative cross-sectional study with cluster analysis based on food intake protocols and nursing documentation. Patients from surgery, internal medicine and university acute geriatric care wards were included in this non-probability sample. Results: Four groups were formed out of the data from 135 patients (protein requirement coverage): Well-nourished (116\%), sufficiently-nourished (77\%), insufficiently-nourished (59\%) and poorlynourished (40\%). A significant correlation between calorie and protein requirement coverage and treatment area has been shown. Conclusions: The degree of coverage of protein- and energy requirement is related to the treatment area and consequently to its team culture and treatment concept. Based on the characteristics of the well-nourished, a positive effect of interprofessional cooperation and systematic recording of the risk of malnutrition, as implemented in the treatment area of acute geriatric care, might be concluded.
\end{abstract}

Keywords: Elderly patients, nursing diagnoses, risk of malnutrition, protein and calorie requirement coverage, acute care hospital 
Was ist zu dieser Thematik schon bekannt?

Die Gefahr einer Mangelernährung ist bei hohem Alter und akuter Erkrankung erhöht.

\section{Was ist neu?}

Die explorative Querschnittsstudie von 135 Fällen zeigte vier Gruppen der Nährstoffzufuhr: Gut-Ernährte, Genügend-Ernährte, Ungenügend-Ernährte und Schlecht-Ernährte.

Welche Konsequenzen haben die Ergebnisse für die Pflegepraxis?

Die Studie zeigt Zusammenhänge von Ernährungszustands-Gruppen zu einem Krankenhausbereich mit interprofessionellem Behandlungskonzept.

\section{Einleitung und Problemstellung}

Diese Studie hat ältere hospitalisierte Personen (80-jährig und älter) im Fokus, da diese infolge hohem Kalorien- und Proteinbedarf eine erhöhte Gefahr für Mangelernährung aufweisen (Volkert et al., 2019). Die Gefahr einer Mangelernährung bei älteren Menschen in Akutspitälern scheint weder systematisch noch umfassend erkannt zu werden, wodurch die notwendigen Interventionen meist unterbleiben. Mangelernährung erhöht die Risiken für Wundinfektionen, akute Verwirrtheit und Stürze und erhöht die Mortalitätsrate (Barker, Gout \& Crowe, 2011). Liegt eine Mangelernährung vor, führt sie folglich zu verlängerten Krankenhausaufenthalten und erhöhten Gesundheitskosten (Barker et al., 2011). Im vorliegenden Forschungsprojekt wird eine leichte Kalorien- und Proteinmangelernährung definiert als

- NRS-Gesamtscore von mindestens 3 [NRS = Nutrition Risk Score 2002 nach Kondrup (Kondrup, Rasmussen, Hamberg, Stanga \& Group, 2003)] und

- ungewollter Gewichtsverlust > $5 \%$ in 3 Monaten oder

- in der vergangenen Woche ungewollt weniger als 50-75\% des Bedarfs zugeführt (Bundesamt für Statistik 2020a, S.200).

Der NRS besteht aus Body Mass Index (BMI, berechnet durch Körpergewicht/Körpergröß $\left.\mathrm{e}^{2}=\mathrm{kg} / \mathrm{m}^{2}\right)$, Alter und Gewichtsverlust in den vorangegangenen drei Monaten sowie Krankheitsschwere. Die Werte des NRS werden wie folgt interpretiert: $0-2$ = tiefes Risiko für Mangelernährung, 3 = Risiko, 4-5 = hohes Risiko für Mangelernährung, wahrscheinlich bereits mangelernährt (Kondrup et al., 2003).

$\mathrm{Zu}$ den Ursachen für Mangelernährung zählen Appetitlosigkeit, altersbedingt verringertes Geschmacksempfinden, enorale Veränderungen und Polypharmazie (Bauer, Halfens \& Lohrmann, 2017). Zudem weisen ältere Menschen vermehrt Kognitionsveränderungen auf, welche Appetit und Nahrungszufuhr vermindern können. Physische Belastungen, beispielsweise durch Schmerzen in den oberen Extremitäten, können die Nahrungsaufnahme zusätzlich limitieren (Barker et al., 2011). Wissenschaftlichen Berichten zu Folge könnten auch die Kultur eines Behand- lungsteams und die Haltung von Pflegefachpersonen einen Einfluss auf den Ernährungszustand der Patient innen während deren Krankenhausaufenthalt haben (Zanini, Bagnasco, Aleo, Timmins \& Sasso, 2017).

Womit die Ernährung der älteren Patient_innen im Akutspital zusammenhängt, ist bislang nur in einzelnen Populationen erforscht (Müller et al., 2017). Es fehlt an aktuellen Studien, welche den Ernährungszustand von älteren Patient_innen in verschiedenen Behandlungsbereichen eines Akutspitals beschreiben und Zusammenhänge mit Vorhandensein und Anzahl von Pflegediagnosen, Anzahl medizinischer Diagnosen und sozialen Faktoren wie Wohnsituation und Alter herstellen. Eine Erhebung solcher Zusammenhänge scheint essenziell, um Risikofaktoren identifizieren zu können, was in einem späteren Projektschritt der Entwicklung von Interventionen dienen soll.

\section{Ziel und Forschungsfrage}

Das Ziel dieser Studie ist, erweiterte Erkenntnisse zum Ernährungszustand älterer hospitalisierter Patient_innen zu gewinnen. Zudem sollen Zusammenhänge zu Pflegediagnosen und Anzahl medizinischer Diagnosen ausgewertet werden. Wenn Zusammenhänge und damit mögliche Patient_innen- oder behandlungsbezogene Risikofaktoren benannt sind, kann das interprofessionelle Behandlungsteam frühzeitig wirksame Interventionen ableiten und umsetzen. Die folgende Forschungsfrage soll beantwortet werden: Welche Muster im Sinne von Zusammenhängen lassen sich in Bezug auf Kalorien- und Proteinbedarfsdeckungsgrade und weitere Patient innen-Merkmale erkennen?

\section{Methode}

\section{Design}

Das Forschungsvorhaben folgt einem explorativen quantitativen Querschnittsdesign gemäß dem Paradigma der quantitativen Sozialforschung (Mayer, 2019). Ein induktives Vorgehen erlaubt es, von den Daten ausgehend ein komplexes Phänomen von verschiedenen Blickwinkeln zu betrachten und damit zusammenhängende Faktoren zu erkennen (Polit \& Beck, 2020). Explorative Studien erkunden ein bislang nur ungenügend erforschtes Themenfeld und beantworten offene Fragen. Mit solchen entdeckenden statistischen Methoden kann nach Mustern im Sinne von Zusammenhängen in einem Datensatz mit vielen Variablen und Fällen gesucht werden (Döring \& Bortz, 2016). Das Vorgehen wird als Querschnittstudie bezeichnet, da während eines Krankenhausaufenthaltes (1 Messzeitpunkt = 5 Tage mit je Frühstück, Mittag- und Abendessen; entspricht 15 Mahlzeiten pro Patient_in) jeweils Mittelwerte der Kalorienbedarfsdeckungsgrade und ein Mittelwert des Proteinbedarfsdeckungsgrades berechnet und für die weiteren Analysen genutzt wurden. 


\section{Setting und Kontext}

Die Datenerhebung fand von September 2018 bis Juni 2019 in einem 200-Betten Akutspital in Zürich (CH) statt. Aufgenommen wurden Patient_innen der allgemeinen Chirurgie (Chirurgie), inneren Medizin (Medizin) und der universitären Klinik für Akutgeriatrie (Akutgeriatrie). Damit wurde ein breites Spektrum an ernährungsbezogenen Daten von Patient_innen mit unterschiedlichsten medizinischen Diagnosen wie auch von verschiedenen Pflegeund Behandlungsteams erfasst. Die Mahlzeiten wurden in der spitaleigenen Küche zubereitet, auf den jeweiligen Pflegestationen erwärmt und durch die Pflegefachpersonen serviert. Es gehörte zu deren Routinetätigkeiten, die Essenstableaus wieder abzuräumen und die Nahrungszufuhr zu dokumentieren.

\section{Stichprobe}

Die Analyse der Essprotokolle von 110 Patient_innen pro Behandlungsbereich wurde als ausreichend große Stichprobe festgelegt, da aufgrund der Theorie der zentralen Tendenz bei einer Stichprobe in dieser Größe eine Normalverteilung sehr wahrscheinlich ist (Schwarz \& Bruderer Enzler, 2018). Übertragbarkeit und Repräsentativität der Daten auf ein vergleichbares Setting und ähnliche hochaltrige Patient_innen könnten mit einer solchen Stichprobengröße möglich werden (Stöcklin, 2010). Um eine Verzerrung der Ergebnisse durch ungleiche Stichproben auszuschließen, wurden gleich viele Patient_innen aus allen drei Behandlungsbereichen eingeschlossen.

Eingeschlossen wurden Daten von allen allgemein versicherten, hospitalisierten 80-jährigen oder älteren Patient innen im vordefinierten Erhebungszeitraum, wenn Essprotokolle von mindestens fünf Tagen vollständig waren, damit je Patient_in verlässliche Durchschnittswerte der Nahrungszufuhr berechnet werden konnten. Dabei wurden je eine Pflegestation der Behandlungsbereiche Chirurgie, Medizin und Akutgeriatrie eingeschlossen. Ausgeschlossen wurden Patient_innen, die Ernährung parenteral oder via Ernährungssonde erhielten und solche, die sich gemäß ärztlichem Bericht in einer terminalen Krankheitssituation befanden.

\section{Datensammlung}

Der Ernährungszustand ist in dieser Studie als Kalorienund Proteinbedarfsdeckungsgrad definiert, wobei grundsätzlich eine 100-prozentige Bedarfsdeckung angestrebt wird (Rosenberger, Rechsteiner, Dietsche \& Breidert, 2019).

Ein interprofessionelles Forschungsteam, bestehend aus einer Advanced Practice Nurse, Ernährungsberaterinnen BSc und Ärzt_innen der Traumatologie, Geriatrie und Medizin, begleiteten diese Studie mit ihrer Expertise zu Mangelernährung und Akutspitalbehandlung.

Für die Erhebung des Ernährungszustandes wurden das Pflegefachpersonal und die Ernährungsberatung mit ei- nem gemeinsam definierten Prozessablauf instruiert. Eine verantwortliche Pflegefachperson kennzeichnete Studienpatient_innen auf der Plantafel gemäß Ein- und Ausschlusskriterien, was durch die operative Projektleitung zweimal wöchentlich überprüft wurde. Die Pflegefachpersonen führten Essprotokolle, indem sie beim Abräumen der Gedecke unmittelbar nach der Mahlzeiteneinnahme bei allen Studienpatient_innen auf den Esskarten protokollierten, welche Menübestandteile (Suppe, Beilage/Stärke, Fleisch / Eiweiß, Gemüse, Dessert) zu einem Viertel, zur Hälfte, zu drei Vierteln, ganz oder gar nicht gegessen wurden. Diese Dokumentation der Zufuhr sowie die Einschätzung der Gefahr der Mangelernährung wird bei der Einführung allen neuen Mitarbeitenden erklärt. Die Ernährungsberatung ermittelte deren Kalorien- und Proteinzufuhr, -bedarf und -deckungsgrade. Die Kalorienund Proteinzufuhr wurde anhand des vordefinierten Nährstoffgehalts der Menüs zusammen mit den Essprotokollen berechnet. Für die Berechnung von Kalorien- und Proteinbedarfsdeckungsgraden (Zufuhr im Verhältnis zum Bedarf) wurden folgende Formeln angewendet: Proteinbedarfsdeckungsgrad in Prozent: 1 Gramm Protein pro Kilogramm Körpergewicht (KG), 1 g / kg KG (Volkert et al., 2019); Kalorienbedarfsdeckungsgrad: 30 Kilokalorien pro Kilogramm Körpergewicht, 30 kcal / kg KG (Volkert et al., 2019) und Kalorienbedarfsdeckungsgrad mit HarrisBenedict-Formel (HB) (Harris \& Benedict, 1919). Da es möglich ist, mehr zu essen als der Körper aufgrund von Berechnungen mit Ernährungsformeln benötigt, können die Grade der Kalorienbedarfs- und Proteinbedarfsdeckung auch mehr als 100 Prozent betragen.

Zudem wurden durch eine Forschungsassistentin folgende weiteren Patient_innen-Merkmale pseudonymisiert in eine Tabelle übertragen: Behandlungsbereich, Geschlecht, Alter, Wohnsituation vor Hospitalisation, Liegedauer in Tagen, NRS, BMI, Anzahl Pflegediagnosen, Vorhandensein einer Pflegediagnose zu Ernährung, Anzahl medizinische Diagnosen, medizinische Hauptdiagnose (ICD-10 Code).

\section{Datenauswertung}

Mit einer Clusteranalyse wurden Muster in den Zusammenhängen von mehreren unabhängigen Variablen zu mehreren abhängigen Variablen ausgewertet (Döring \& Bortz, 2016). Damit sollen Erkenntnisse über das komplexe Phänomen „Gefahr einer Mangelernährung“ gewonnen werden. Die drei Hauptschritte des ClusteranalyseVerfahrens sind anbei beschrieben.

Im ersten Schritt der Datenanalyse wurden Häufigkeitsverteilungen und Mittelwerte bestimmt. Schiefe beziehungsweise Kurtosis weisen auf Normalverteilung hin, sofern die Werte zwischen 0 und 3 beziehungsweise 0 und 2 sind (Hager, 2019). Zum vereinfachten, übersichtlichen Vergleich wurden unabhängige Variablen dichotomisiert oder in drei Kategorien aufgeteilt, je nach theoriegeleiteter Sinnhaftigkeit. So wurde beispielsweise die 
Variable BMI in die zwei Kategorien „BMI $<24 \mathrm{~kg} / \mathrm{m}^{2 \text { “ }}$ oder „BMI $\geq 24 \mathrm{~kg} / \mathrm{m}^{2 \text { " }}$ unterteilt, da der Norm-BMI bei älteren Patient_innen 24 bis $31 \mathrm{~kg} / \mathrm{m}^{2}$ beträgt (Rosenberger et al., 2019).

Im zweiten Schritt wurden Gruppen gebildet mit Fällen, welche einen ähnlichen Protein- und Kalorienbedarfsdeckungsgrad aufwiesen. Dazu wurde die hierarchische Clusteranalyse mit Ward-Verfahren gewählt, was der gängigsten Methode für intervallskalierte Daten entspricht (Schwarz \& Bruderer Enzler, 2018; Stein \& Vollnhals, 2011). Die Absicht für geeignete Gruppen war, dass die Patient_innen innerhalb einer Gruppe bezüglich der beiden Variablen zu Kalorienbedarfsdeckungsgraden und dem Proteinbedarfsdeckungsgrad sehr ähnlich sein sollten. Zugleich unterscheiden sich die verschiedenen Gruppen hinsichtlich dieser Deckungsgrade bedeutsam.

Die Unähnlichkeitsberechnung erfolgte mit standardisierten Daten (0-1) und anschließender Bestimmung der euklidischen Distanz mit SPSS 26 (IBM, 2020). Damit sollen typische Patient_innen-Gruppen aufgefunden werden. Die Gruppengrößen sollten zudem ähnlich und mit zwei bis sechs Gruppen übersichtlich sein. Im dritten Schritt wurden Eigenschaften je Gruppe exploriert und beschrieben. Zur genaueren Überprüfung von möglichen Zusammenhängen aufgrund der Clusteranalyse wurde zusätzlich eine Regressionsanalyse durchgeführt.

Zusammenhänge zwischen Clustereigenschaften und Patient_innen-Merkmalen wurden im Forschungsteam ermittelt und in der Diskussion mit einer Statistikerin zur Minimierung des Verzerrungsrisikos überprüft. Die Qualität der Berichterstattung wurde gemäß STROBE für quantitative Beobachtungsstudien sichergestellt (von Elm et al., 2008).

\section{Ethische Aspekte}

Diese Studie wurde mit Routinedaten als Teil eines Qualitätssicherungsprojektes durchgeführt und daher als ethisch unbedenklich erklärt (Req-2016-00670). Die Projektdurchführung hält sich an die Deklaration von Helsinki (Hill, Subchaturas \& Kloiber, 2013), die internationalen
Good Clinical Practice Guidelines (ICH, 2016) sowie das lokal geltende Schweizer Gesetz (Bundesgesetz über die Forschung am Menschen (Humanforschungsgesetz)) und die kantonalen ethischen Grundsätze (Kantonale Ethikkommission Zürich, 2017). Die Einwilligung der Institution liegt in Form des Projektauftrages vor. Gesundheitsbezogene Personendaten wurden vor Beginn der Analyse pseudonymisiert, sodass keine Rückschlüsse auf einzelne Personen möglich sind (kofam, 2017). Alle physischen Patientendaten wurden in einem Schrank eingeschlossen und digitale Daten verschlüsselt aufbewahrt.

\section{Ergebnisse}

\section{Beschreibung der Stichprobe}

In der Halbzeit der geplanten Datensammlung waren je Bereich 18-38 der geforderten 110 Patientenfälle eingeschlossen. Die beiden Supervisorinnen und Auftraggeber entschieden zusammen mit dem Forschungsteam, die Zielgröße von 110 Fällen auf je 45 Fälle pro Behandlungsbereich zu reduzieren, damit der Forschungszeitplan eingehalten werden konnte. Zudem blieb die Priorität, von jedem Bereich gleich viele Patient_innen einzubeziehen, um eine Verzerrung aufgrund der Ungleichverteilung der Anzahl Fälle je Bereich zu vermeiden. Es wurden somit die jeweils ersten 45 Patient_innen je Behandlungsbereich eingeschlossen ( $\mathrm{N}=135)$, was der Berechnung von 2025 Essprotokollen (fünf Tage à drei Mahlzeiten von 45 Patient_innen) entspricht. Wie in den Tabellen 1 und 2 aufgeführt, wurden 87 (64\%) Frauen und 48 (36\%) Männer mit einem Durchschnittsalter von 86 Jahren eingeschlossen. Von 213 älteren Patient_innen wurden 35 ausgeschlossen, da sie weniger als fünf Tage hospitalisiert waren, 27 infolge unvollständiger Essprotokolle, neun wegen terminalem Krankheitszustand und sieben, da sie parenteral ernährt wurden.

Bei den eingeschlossenen Patient_innen waren keine bis sieben Pflegediagnosen erfasst. Die drei häufigsten medizinischen Diagnosen betrafen das Atmungssystem, Muskel-Skelett-System und Urogenitalsystem.

Tabelle 1. Beschreibung der Stichprobe mit Ein- und Ausschlussgründen

\begin{tabular}{|c|c|c|c|c|}
\hline Gründe für Patient_innenausschluss & Total & Akutgeriatrie & Chirurgie & Innere Medizin \\
\hline im Erhebungszeitraum z 80-jährig (Jahrgang 1938/1939 und tiefer) & 213 & 63 & 63 & 87 \\
\hline Ausgeschlossen Anzahl & 78 & 18 & 18 & 42 \\
\hline \multicolumn{5}{|l|}{ Gründe: } \\
\hline zu kurze Liegedauer & 35 & 0 & 8 & 27 \\
\hline nicht drei vollständige Esskarten von 5 Tagen & 27 & 13 & 6 & 8 \\
\hline terminal/während dieser Hospitalisation verstorben & 9 & 3 & 2 & 4 \\
\hline parenteral ernährt & 7 & 2 & 2 & 3 \\
\hline Eingeschlossen & 135 & 45 & 45 & 45 \\
\hline männlich & $48(36 \%)$ & 8 & 21 & 19 \\
\hline weiblich & $87(64 \%)$ & 37 & 24 & 26 \\
\hline
\end{tabular}


Tabelle 2. Protein- und Kalorienbedarfsdeckungsgrad je Behandlungsbereich

\begin{tabular}{|c|c|c|c|c|c|}
\hline \multicolumn{3}{|l|}{ Patient_innenmerkmale/Behandlungsbereich } & \multirow{2}{*}{$\begin{array}{c}\text { Akutgeriatrie } \\
98,86\end{array}$} & \multirow{2}{*}{$\begin{array}{c}\text { Chirurgie } \\
66,98\end{array}$} & \multirow{2}{*}{$\begin{array}{c}\text { Innere Medizin } \\
67,57\end{array}$} \\
\hline $\begin{array}{l}\text { Deckungsgrad in Prozent für Kalorienbedarf } \\
\text { gemäß Harris Benedict Formel }\end{array}$ & Mittelwert & & & & \\
\hline $\begin{array}{l}\text { Deckungsgrad in Prozent für Proteinbedarf ge } \\
\text { mäß } 1 \mathrm{~g} / \mathrm{kgKG}\end{array}$ & - Mittelwert & & 92,82 & 69,00 & 61,38 \\
\hline $\begin{array}{l}\text { Deckungsgrad in Prozent für } \\
\text { Kalorienbedarf gemäss } 30 \mathrm{kcal} / \mathrm{kgKG}\end{array}$ & Mittelwert & & 88,82 & 60,48 & 58,44 \\
\hline Body Mass Index (kg / m²) & Mittelwert & & 24,28 & 25,15 & 25,35 \\
\hline Alter (Jahren) & Mittelwert & & 87 & 84 & 87 \\
\hline \multirow[t]{9}{*}{ Wohnsituation } & zu Hause & Anzahl & 31 & 34 & 35 \\
\hline & & Anzahl als Zeilen (\%) & $31,0 \%$ & $34,0 \%$ & $35,0 \%$ \\
\hline & & Anzahl als Spalten (\%) & $68,9 \%$ & $75,6 \%$ & $77,8 \%$ \\
\hline & Alterswohnung & Anzahl & 3 & 3 & 7 \\
\hline & & Anzahl als Zeilen (\%) & $23,1 \%$ & $23,1 \%$ & $53,8 \%$ \\
\hline & & Anzahl als Spalten (\%) & $6,7 \%$ & $6,7 \%$ & $15,6 \%$ \\
\hline & Pflegezentrum & Anzahl & 11 & 8 & 3 \\
\hline & & Anzahl als Zeilen (\%) & $50,0 \%$ & $36,4 \%$ & $13,6 \%$ \\
\hline & & Anzahl als Spalten (\%) & $24,4 \%$ & $17,8 \%$ & $6,7 \%$ \\
\hline \multirow[t]{6}{*}{ Geschlecht } & männlich & Anzahl & 8 & 21 & 19 \\
\hline & & Anzahl als Zeilen (\%) & $16,7 \%$ & $43,8 \%$ & $39,6 \%$ \\
\hline & & Anzahl als Spalten (\%) & $17,8 \%$ & $46,7 \%$ & $42,2 \%$ \\
\hline & weiblich & Anzahl & 37 & 24 & 26 \\
\hline & & Anzahl als Zeilen (\%) & $42,5 \%$ & $27,6 \%$ & $29,9 \%$ \\
\hline & & Anzahl als Spalten (\%) & $82,2 \%$ & $53,3 \%$ & $57,8 \%$ \\
\hline \multirow[t]{18}{*}{ Nutrition Risk Score } & 1 & Anzahl & $0 \%$ & 2 & 3 \\
\hline & & Anzahl als Zeilen (\%) & $0 \%$ & $40,0 \%$ & $60,0 \%$ \\
\hline & & Anzahl als Spalten (\%) & $0 \%$ & $4,4 \%$ & $6,7 \%$ \\
\hline & 2 & Anzahl & 11 & 16 & 8 \\
\hline & & Anzahl als Zeilen (\%) & $31,4 \%$ & $45,7 \%$ & $22,9 \%$ \\
\hline & & Anzahl als Spalten (\%) & $24,4 \%$ & $35,6 \%$ & $17,8 \%$ \\
\hline & 3 & Anzahl & 14 & 11 & 20 \\
\hline & & Anzahl als Zeilen (\%) & $31,1 \%$ & $24,4 \%$ & $44,4 \%$ \\
\hline & & Anzahl als Spalten (\%) & $31,1 \%$ & $24,4 \%$ & $44,4 \%$ \\
\hline & 4 & Anzahl & 14 & 8 & 7 \\
\hline & & Anzahl als Zeilen (\%) & $48,3 \%$ & $27,6 \%$ & $24,1 \%$ \\
\hline & & Anzahl als Spalten (\%) & $31,1 \%$ & $17,8 \%$ & $15,6 \%$ \\
\hline & 5 & Anzahl & 3 & 7 & 7 \\
\hline & & Anzahl als Zeilen (\%) & $17,6 \%$ & $41,2 \%$ & $41,2 \%$ \\
\hline & & Anzahl als Spalten (\%) & $6,7 \%$ & $15,6 \%$ & $15,6 \%$ \\
\hline & 6 & Anzahl & 3 & 1 & 0 \\
\hline & & Anzahl als Zeilen (\%) & $75,0 \%$ & $25,0 \%$ & $0 \%$ \\
\hline & & Anzahl als Spalten (\%) & $6,7 \%$ & $2,2 \%$ & $0 \%$ \\
\hline Liegedauer & Mittelwert & & 13 & 17 & 15 \\
\hline Anzahl medizinischer Diagnosen & Mittelwert & & 10 & 6 & 6 \\
\hline Anzahl Pflegediagnosen & Mittelwert & & 3 & 3 & 3 \\
\hline \multirow{6}{*}{$\begin{array}{l}\text { Vorhandensein einer Pflegediagnose } \\
\text { zu Mangelernährung, Ernährung } \\
\text { als Intervention }\end{array}$} & nein & & 19 & 18 & 35 \\
\hline & & Anzahl als Zeilen (\%) & $26,4 \%$ & $25,0 \%$ & $48,6 \%$ \\
\hline & & Anzahl als Spalten (\%) & $42,2 \%$ & $40,0 \%$ & $77,8 \%$ \\
\hline & ja & Anzahl & 26 & 27 & 10 \\
\hline & & Anzahl als Zeilen (\%) & $41,3 \%$ & $42,9 \%$ & $15,9 \%$ \\
\hline & & Anzahl als Spalten (\%) & $57,8 \%$ & $60,0 \%$ & $22,2 \%$ \\
\hline
\end{tabular}

Anmerkungen: $\mathrm{g} / \mathrm{kgKG}=$ Gramm pro Kilogramm Körpergewicht; $\mathrm{kcal}=$ Kilokalorien, $\mathrm{kg} / \mathrm{m}^{2}$ = Körpergewicht in Kilogramm dividiert durch Größe in Meter hoch zwei. 


\section{Häufigkeitsverteilung und Mittelwerte}

Tabelle 3 zeigt die Ergebnisse der beschreibenden statistischen Datenauswertung der beiden Variablen zu Kalorienbedarfsdeckungsgraden und dem Proteinbedarfsdeckungsgrad mit Mittelwerten von 69-78\%, welche gemäß Kolmogorov-Smirnov und Shapiro-Wilk Test nicht normalverteilt sind (siehe Elektronisches Supplement ESM1: Tabelle S1). Der durchschnittliche BMI der eingeschlossenen Patient_innen beträgt $25 \mathrm{~kg} / \mathrm{m}^{2}$ mit einer Spanne von 15 bis $42 \mathrm{~kg} / \mathrm{m}^{2}$. Die Histogramme sind in den Abbildungen $1-4$ des ESM1 gezeigt. Ein NRS $\geq 3$ war bei $70 \%$ der Gesamtstichprobe dokumentiert, was auf eine Gefahr für Mangelernährung hinweist (ESM1, Tabelle S2).

Die Werte der Kalorienbedarfsdeckungsgrade berechnet mit $\mathrm{HB}$ und $30 \mathrm{kcal} / \mathrm{kg} \mathrm{KG}$ und der Proteinbedarfsdeckungsgrad $1 \mathrm{~g} / \mathrm{kg}$ KG korrelieren signifikant mit Korrelationskoeffizienten von $>0,8(p<0,001)$, wie in Tabelle 4 dargestellt.

\section{Gruppenbildung durch Clusteranalyse}

Aufgrund der Ähnlichkeiten und Anzahl der Patient_innen wurden anhand des Dendrogramms (siehe ESM2) vier Cluster (Gruppen) gebildet. Diese wurden als Gut-Ernährte ( $\mathrm{n}=35)$, Genügend-Ernährte $(\mathrm{n}=34)$, Ungenügend-Ernährte $(n=39)$ und Schlecht-Ernährte $(n=27)$ benannt, wobeisieeinendurchschnittlichenProteinbedarfsdeckungsgrad von rund $116 \%, 77 \%$, 59\% und $40 \%$ aufwiesen. Ernährungszustand mit Bedarfsdeckungsgrad sowie demografische Angaben und die Anzahl Pflegediagnosen und medizinischen Diagnosen sind in Tabelle 5 dargestellt.
Wird die Tabelle 5 zusammengefasst, so kann hervorgehoben werden, dass von der Gruppe „Gut-Ernährte“ der größte Teil (76\%) in der Akutgeriatrie behandelt war, während der Anteil dieses Behandlungsbereiches in der Gruppe der „Schlecht-Ernährten“ nur 11\% ausmachte. Ein Drittel dieser Gruppe waren 90-jährig und älter, der Frauenanteil betrug $77 \%$. Während $33 \%$ der „Schlecht-Ernährten“ im Pflegezentrum lebten, waren es bei den „GutErnährten" $14 \%$. Ein BMI $<24 \mathrm{~kg} / \mathrm{m}^{2}$ wurde bei $79 \%$ der „Gut-Ernährten“ gemessen. Bei 85 \% dieser Patient_innen wurde ein NRS $\geq 3$ und bei 16 von 35 Fällen wurde eine explizite Pflegediagnose im Zusammenhang mit Ernährung (Mangelernährung, Übelkeit, dysfunktionale gastrointestinale Motilität) festgehalten. Drei oder mehr Pflegediagnosen waren bei $60 \%$ der „Gut-Ernährten“ dokumentiert.

Auf der Akutgeriatrie wird ein interprofessionelles Behandlungskonzept gelebt. Unter anderem gehört dazu ein wöchentlicher interdisziplinärer Rapport, bei dem das Pflegefachpersonal sowie jeder therapeutische Dienst das Behandlungsziel definiert und evaluiert. Alle Patient_innen haben in diesem Bereich Ernährungstherapie (Stadtspital Waid und Triemli, 2020). Im Vergleich dazu waren 89\% aller Fälle der „Schlecht-Ernährten“ auf Chirurgie und Medizin, wo scheinbar die Ernährungstherapie bei diesen älteren Patient_innen einen geringeren Stellenwert hat. Dieser Umstand deutet darauf hin, dass die Teamkultur und das Bewusstsein für die Wichtigkeit der Patientenernährung eines Behandlungsbereich den Ernährungszustand mitbeeinflussen.

Zusammenhänge zwischen Ernährungszustand im Sinne des Kalorien- und Proteinbedarfsdeckungsgrades und der Anzahl Pflegediagnosen oder dem Vorhandensein einer ernährungsbezogenen Pflegediagnose wurden nicht statis-

Tabelle 3. Verteilungsform der metrischen Variablen

\begin{tabular}{|c|c|c|c|c|c|c|c|c|c|}
\hline & \multirow{2}{*}{$\begin{array}{c}\mathrm{N} \\
\text { Statistik }\end{array}$} & \multirow{2}{*}{$\begin{array}{l}\text { Minimum } \\
\text { Statistik }\end{array}$} & \multirow{2}{*}{$\begin{array}{l}\text { Maximum } \\
\text { Statistik }\end{array}$} & \multirow{2}{*}{$\begin{array}{l}\text { Mittelwert } \\
\text { Statistik }\end{array}$} & \multirow{2}{*}{$\begin{array}{c}\text { Standard- } \\
\text { Abweichung } \\
\text { Statistik }\end{array}$} & \multicolumn{2}{|c|}{ Schiefe } & \multicolumn{2}{|c|}{ Kurtosis } \\
\hline & & & & & & Statistik & $\begin{array}{l}\text { Standard- } \\
\text { fehler }\end{array}$ & Statistik & $\begin{array}{l}\text { Standard- } \\
\text { fehler }\end{array}$ \\
\hline Body Mass Index (kg / m²) & 135 & 15,05 & 42,38 & 24,92 & 5,03 & 0,66 & 0,21 & 0,75 & 0,41 \\
\hline Alter & 135 & 80 & 98 & 86 (Median) & 4,23 & 0,43 & 0,21 & $-0,60$ & 0,41 \\
\hline $\begin{array}{l}\text { Deckungsgrad in Prozent } \\
\text { für Energiebedarf gemäß } \\
\text { Harris Benedict Formel }\end{array}$ & 135 & 19,49 & 166,22 & 77,80 & 28,80 & 0,53 & 0,21 & 0,00 & 0,41 \\
\hline $\begin{array}{l}\text { Deckungsgrad in Prozent } \\
\text { für Proteinbedarf gemäß } \\
1 \mathrm{~g} / \mathrm{kgKG}\end{array}$ & 135 & 13,43 & 188,89 & 74,40 & 31,99 & 0,87 & 0,21 & 1,19 & 0,41 \\
\hline $\begin{array}{l}\text { Deckungsgrad in Prozent } \\
\text { für Energiebedarf gemäß } \\
30 \text { kcal / kgKG }\end{array}$ & 135 & 16,39 & 161,56 & 69,24 & 28,53 & 0,90 & 0,21 & 0,80 & 0,41 \\
\hline Liegedauer & 135 & 7 & 64 & 13 (Median) & 9,84 & 3,06 & 0,21 & 10,84 & 0,41 \\
\hline $\begin{array}{l}\text { Anzahl medizinischer } \\
\text { Diagnosen }\end{array}$ & 135 & 1 & 14 & 7 (Median) & 3,17 & 0,07 & 0,21 & $-0,60$ & 0,41 \\
\hline Anzahl Pflegediagnosen & 135 & 0 & 7 & 3 (Median) & 1,53 & 0,52 & 0,21 & $-0,22$ & 0,41 \\
\hline Gültige Werte (Listenweise) & 135 & & & & & & & & \\
\hline
\end{tabular}

Anmerkungen: g / kgKG = Gramm pro Kilogramm Körpergewicht; kcal = Kilokalorien, kg / m² = Körpergewicht in Kilogramm dividiert durch quadrierte Größe in Metern. 
tisch bestätigt (Tabelle 6). Zur genaueren Beschreibung der vier Gruppen wurden Unterschiede zwischen den Gruppen mit Chi-Quadrat nach Pearson bestimmt, da die Variablen intervallskaliert oder ordinalskaliert waren. Die vier Gruppen unterscheiden sich signifikant bezüglich der Kalorien- und Proteinbedarfsdeckungsgrade und der Anteile je Gruppe aus den verschiedenen Behandlungsbereichen. Der Anteil an Patient_innen aus der Akutgeriatrie war signifikant höher in der Gruppe der „Gut-Ernährten“ als in den anderen Gruppen. Zudem war der Anteil an Patient_innen mit BMI $<24 \mathrm{~kg} / \mathrm{m}^{2}$ der „Gut-Ernährten“ signifikant größer als in den anderen drei Gruppen, während die anderen drei Gruppen einen signifikant höheren Anteil Patient_innen mit $\mathrm{BMI} \geq 24 \mathrm{~kg} / \mathrm{m}^{2}$ aufwiesen.

Anhand eines generalisierten Regressionsmodells mit Gamma-Verteilung (da nicht-normalverteilte Daten) mit jeder Variable des Kalorien- und Proteinbedarfsdeckungsgrades wurde ebenfalls aufgezeigt, dass der Bedarfsdeckungsgrad signifikant mit dem Behandlungsbereich zusammenhängt. Bei den eingeschlossenen 45 Fällen pro Bereich konnten keine statistisch signifikanten Zusammenhänge zu Anzahl medizinischer und Anzahl pflegerischer Diagnosen gezeigt werden (siehe ESM1: Tabellen S3-S17).

\section{Diskussion}

Erstmalig wurde im deutschsprachigen Akutspital-Kontext ein Zusammenhang von Nahrungszufuhr zu Pflegediagnosen wie zur Anzahl medizinischer Diagnosen und weiteren Merkmalen von älteren Patient_innen untersucht. Signifikante Zusammenhänge zeigten sich von Ernährungszustands-Gruppe $\mathrm{zu}$ Behandlungsbereich und zu BMI-Kategorie. Aufgrund des jeweiligen Anteils Fälle je Bereich in den verschiedenen Gruppen könnte vermutet werden, dass die Gefahr für Mangelernährung nicht in allen Behandlungsbereichen berücksichtigt wurde. Diese Annahme basiert auf der Tatsache, dass $50 \%$ aller Fälle der „Gut Ernährten“ einen NRS > 3 aufwiesen. Es kann vermutet werden, dass bei diesen Patient_innen die Gefahr für Mangelernährung erkannt wurde, was zu individueller Ernährungstherapie, gezielten Pflegeinterventionen und dadurch einem hohen Bedarfsdeckungsgrad führte. Die Wirksamkeit der Ernährungstherapie während des Krankenhausaufenthaltes ist mehrfach beschrieben (Gomes et al., 2019).

In der Gruppe „Ungenügend Ernährte“ waren $95 \%$ der Patient_innen von den Behandlungsbereichen Medizin und Chirurgie. Diese Ergebnisse lassen vermuten, dass das Bewusstsein eines Behandlungsteams die Kalorien- und Proteinbedarfsdeckungsgrade der älteren Patient_innen im Akutspital beeinflussen. Die Auswirkungen einer ernährungsfokussierten Teamkultur sowie die Haltung der Pflegefachpersonen wurden sowohl in einer Querschnittsstudie (Vanderwee et al., 2011), wie auch in 
einem fundierten Editorial (Zanini et al., 2017) erklärt. Tappenden et al. (2013) haben zudem die Bildung einer Teamkultur, welche die Ernährung als Therapiebestandteil berücksichtigt, als wichtige Intervention, um Mangelernährung vorzubeugen, beschrieben. Der Einfluss der Teamkultur und das Bewusstsein für die Wichtigkeit der Patientenernährung wurden auch im subakuten Klinikbereich beobachtet (Ottrey, Porter, Huggins \& Palermo, 2018). Die Kalorien- und Proteinbedarfsdeckungsgrade von älteren Patient_innen anderer Bereiche wie Medizin und Chirurgie könnte durch ein interprofessionelles Behandlungskonzept mit systematischer Einschätzung der Gefahr einer Mangelernährung verbessert werden (Beck, Geser \& Grob, 2012).
Mögliche Gründe für das Fehlen eines statistischen $\mathrm{Zu}$ sammenhanges zwischen der Anzahl Pflegediagnosen und der Gruppenzugehörigkeit könnten die relativ wenigen Patient_innen je Gruppe sowie die geringe Menge an Pflegediagnosen je Patient_in sein. Aus Diskussionen der Datenanalyse innerhalb des Forschungsteams geht jedoch hervor, dass teilweise Syndrom-Pflegediagnosen erstellt wurden, was auch bei komplexen Patientensituationen zu einer geringen Anzahl Pflegediagnosen führte.

Bei der Ergebnisdiskussion tauchte die Frage auf, weshalb die Gruppe der "Gut-Ernährten“ einen signifikant höheren Anteil an Patient_innen mit BMI $<24 \mathrm{~kg} / \mathrm{m}^{2}$ aufweist als die anderen Gruppen. Es gibt einerseits die mathemati-

Tabelle 5. Gruppen (Cluster) von ähnlichen Patient_innenfällen in Bezug auf Kalorien- und Proteinbedarfsdeckungsgrad

\begin{tabular}{|c|c|c|c|c|c|c|}
\hline 4 Gruppen (Nährstoffzufuhr) & & Gut-Ernährte & Genügend-Ernährte & Ungenügend Ernährte & Schlecht-Ernährte & Gesamt \\
\hline Gruppengröße n (\%) & Anzahl & $35(25,9 \%)$ & $34(25,2 \%$ & $39(28,9 \%)$ & $27(20,0 \%)$ & $135(100,0 \%)$ \\
\hline $\begin{array}{l}\text { Proteinbedarfsdeckungsgrad } \\
1 \mathrm{gr} / \mathrm{kg} \text { in Prozent }\end{array}$ & $\begin{array}{l}\text { Mittelwert } \\
\text { Min-Max }\end{array}$ & $\begin{array}{c}115,8 \% \\
(82-189 \%)\end{array}$ & $\begin{array}{c}76,6 \% \\
(55-104 \%\end{array}$ & $\begin{array}{c}58,9 \% \\
(30-81 \%)\end{array}$ & $\begin{array}{c}40,4 \% \\
(13-76 \%)\end{array}$ & $\begin{array}{c}74,4 \% \\
(13-189 \%)\end{array}$ \\
\hline $\begin{array}{l}\text { Kalorienbedarfsdeckungsgrad } \\
30 \text { kcal / kg in Prozent }\end{array}$ & $\begin{array}{l}\text { Mittelwert } \\
\text { Min-Max }\end{array}$ & $\begin{array}{c}107,8 \% \\
(81-162 \%)\end{array}$ & $\begin{array}{c}71,9 \% \\
(64-95 \%)\end{array}$ & $\begin{array}{c}55,1 \% \\
(49-66 \%)\end{array}$ & $\begin{array}{c}36,4 \% \\
(19-56 \%)\end{array}$ & $\begin{array}{c}69,2 \% \\
(16-162 \%)\end{array}$ \\
\hline $\begin{array}{l}\text { Kalorienbedarfsdeckungsgrad } \\
\text { HB in Prozent }\end{array}$ & $\begin{array}{l}\text { Mittelwert } \\
\text { Min-Max }\end{array}$ & $\begin{array}{c}116,0 \% \\
(90-166 \%)\end{array}$ & $\begin{array}{c}83,5 \% \\
(67-97 \%)\end{array}$ & $\begin{array}{c}63,1 \% \\
(52-76 \%)\end{array}$ & $\begin{array}{c}42,3 \% \\
(16-51 \%)\end{array}$ & $\begin{array}{c}77,8 \% \\
(20-162 \%)\end{array}$ \\
\hline Bereich & Akutgeriatrie & $66 \%$ & $47 \%$ & $5 \%$ & $15 \%$ & $33 \%$ \\
\hline \multirow[t]{2}{*}{ (Spaltenprozent§) } & Chirurgie & $14 \%$ & $29 \%$ & $49 \%$ & $41 \%$ & $33 \%$ \\
\hline & Innere Medizin & $20 \%$ & $24 \%$ & $46 \%$ & $44 \%$ & $33 \%$ \\
\hline \multirow[t]{2}{*}{ Geschlecht } & Männlich & $23 \%$ & $35 \%$ & $41 \%$ & $44 \%$ & $36 \%$ \\
\hline & Weiblich & $77 \%$ & $65 \%$ & $59 \%$ & $56 \%$ & $64 \%$ \\
\hline Alter in Jahren§ & $\begin{array}{c}80-84 \\
85-89 \\
90 \text { und älter }\end{array}$ & $\begin{array}{l}37 \% \\
26 \% \\
37 \%\end{array}$ & $\begin{array}{l}32 \% \\
44 \% \\
24 \%\end{array}$ & $\begin{array}{l}49 \% \\
39 \% \\
13 \%\end{array}$ & $\begin{array}{l}48 \% \\
33 \% \\
19 \%\end{array}$ & $\begin{array}{l}42 \% \\
36 \% \\
23 \%\end{array}$ \\
\hline \multirow[t]{2}{*}{$\begin{array}{l}\text { Wohnsituation vor } \\
\text { Hospitalisation }\end{array}$} & $\begin{array}{c}\text { zu Hause/ } \\
\text { Alterswohnung }\end{array}$ & $86 \%$ & $88 \%$ & $90 \%$ & $67 \%$ & $84 \%$ \\
\hline & Pflegezentrum & $14 \%$ & $12 \%$ & $10 \%$ & $33 \%$ & $16 \%$ \\
\hline \multirow[t]{3}{*}{ Liegedauer } & 7 - 10 Tage & $29 \%$ & $44 \%$ & $33 \%$ & $19 \%$ & $32 \%$ \\
\hline & $11-15$ Tage & $46 \%$ & $29 \%$ & $33 \%$ & $41 \%$ & $37 \%$ \\
\hline & $16-64$ Tage & $26 \%$ & $26 \%$ & $33 \%$ & $41 \%$ & $31 \%$ \\
\hline \multirow[t]{3}{*}{ NRS-2002§ } & NRS $0-2$ & $14 \%$ & $38 \%$ & $41 \%$ & $22 \%$ & $30 \%$ \\
\hline & NRS 3 & $34 \%$ & $32 \%$ & $41 \%$ & $22 \%$ & $33 \%$ \\
\hline & NRS $4-5$ & $51 \%$ & $29 \%$ & $18 \%$ & $56 \%$ & $37 \%$ \\
\hline \multirow[t]{2}{*}{ BMI dichotomisiert§ } & $<24 \mathrm{~kg} / \mathrm{m}^{2}$ & $79 \%$ & $44 \%$ & $39 \%$ & $33 \%$ & $49 \%$ \\
\hline & $\geq 24 \mathrm{~kg} / \mathrm{m}^{2}$ & $21 \%$ & $56 \%$ & $62 \%$ & $67 \%$ & $51 \%$ \\
\hline \multirow[t]{4}{*}{ Anzahl Pflegediagnosen§ } & $0-2$ & $40 \%$ & $44 \%$ & $49 \%$ & $33 \%$ & $42 \%$ \\
\hline & $3-4$ & $43 \%$ & $44 \%$ & $39 \%$ & $41 \%$ & $42 \%$ \\
\hline & $>4$ & $17 \%$ & $12 \%$ & $13 \%$ & $26 \%$ & $16 \%$ \\
\hline & Mittelwert & 2,9 & 2,9 & 2,6 & 3,5 & 3,0 \\
\hline Pflegediagnose zu Ernährung§ & vorhanden & $46 \%$ & $53 \%$ & $31 \%$ & $56 \%$ & $45 \%$ \\
\hline \multirow{3}{*}{$\begin{array}{l}\text { Anzahl medizinischer } \\
\text { Diagnosen§ }\end{array}$} & $<6$ & $31 \%$ & $21 \%$ & $46 \%$ & $22 \%$ & $31 \%$ \\
\hline & $6-8$ & $29 \%$ & $38 \%$ & $33 \%$ & $52 \%$ & $37 \%$ \\
\hline & $>8$ & $40 \%$ & $41 \%$ & $21 \%$ & $26 \%$ & $32 \%$ \\
\hline Anzahl Medizinische Diagnosen & Mittelwert & 7,4 & 8,0 & 5,8 & 7,0 & 7,0 \\
\hline
\end{tabular}

Anmerkungen: $\S=$ prozentualer Anteil Fälle je Gruppe, auf ganze Zahlen gerundet, Min - Max: kleinster und größter Mittelwert je Cluster; HB = Harris Benedict, BMI = Body Mass Index, NRS-2002 = Nutrition Risk Score 2002 nach Kondrup; g / kgKG = Gramm pro Kilogramm Körpergewicht; kcal = Kilokalorien, $\mathrm{kg} / \mathrm{m}^{2}=$ Körpergewicht in Kilogramm dividiert durch Größe in Meter hoch zwei. 
sche Begründung, da derselbe Parameter (kg KG) für die Bestimmung des BMI wie auch der Protein- und Kalorienbedarfsberechnung und entsprechend für die Berechnung des Bedarfsdeckungsgrades genutzt wird. Gemäß diesen Formeln haben Patient_innen mit tieferem BMI (meist mit tieferem Gewicht verknüpft) auch einen tieferen Kalorienbedarf. Zudem wurde im deutschsprachig-europäischen Kontext der mit Harris-Benedict-Formel errechnete Kalorien-Bedarf von älteren akut erkrankten Patient_innen nur von 1,5\% der Stichprobe gedeckt (Rosenberger et al., 2019).

Andererseits ist auch vorstellbar, dass ein tiefer BMI für das Behandlungsteam eine offensichtliche Gefahr einer
Mangelernährung darstellt. Diese Annahme ist gestützt auf die Tatsache, dass bei rund $60 \%$ der Patient_innen mit $\mathrm{BMI}<24 \mathrm{~kg} / \mathrm{m}^{2}$ ein NRS von 4-5 und eine ernährungsspezifische Pflegediagnose mit ernährungsfördernden Interventionen dokumentiert war. Umgekehrt formuliert, ergriffen vermutlich Pflegefachpersonen mehrheitlich Maßnahmen, um die Patientenernährung zu optimieren in jenen Fällen, wo die Gefahr einer Mangelernährung bei Eintritt mit NRS und Pflegediagnose korrekt erkannt wurde. Dies hatte anscheinend einen genügenden bis guten Ernährungsbedarfsdeckungsgrad während des Krankenhausaufenthaltes zur Folge.

Tabelle 6. Signifikanztests mit Pearson Chi-Quadrat der vier Cluster, Vergleiche der Spaltenanteile

\begin{tabular}{|c|c|c|c|c|c|}
\hline & & Gut-Ernährte (A) & $\begin{array}{l}\text { Genügend- } \\
\text { Ernährte (B) }\end{array}$ & $\begin{array}{l}\text { Ungenügend- } \\
\text { Ernährte (C) }\end{array}$ & $\begin{array}{l}\text { Schlecht- } \\
\text { Ernährte (D) }\end{array}$ \\
\hline \multirow[t]{2}{*}{ Behandlungsbereich } & Akutgeriatrie & $\begin{array}{l}C(, 000) \\
D(, 000)\end{array}$ & $\begin{array}{l}C(, 000) \\
D(, 046)\end{array}$ & & \\
\hline & $\begin{array}{l}\text { Chirurgie } \\
\text { innere Medizin }\end{array}$ & & & $A(, 009)$ & \\
\hline Geschlecht & $\begin{array}{l}\text { männlich } \\
\text { weiblich }\end{array}$ & & & & \\
\hline \multirow[t]{3}{*}{ Alter } & $80-84$ & & & & \\
\hline & $85-89$ & & & & \\
\hline & 90 und älter & & & & \\
\hline \multirow[t]{2}{*}{ Wohnsituation vor Hospitalisation } & zu Hause / Alterswohnung & & & & \\
\hline & Pflegezentrum & & & & \\
\hline \multirow[t]{3}{*}{ Liegedauer } & relativ kurz $(7-10)$ & & & & \\
\hline & normal $(11-15)$ & & & & \\
\hline & lange $(16-64)$ & & & & \\
\hline \multirow[t]{3}{*}{ Nutrition Risk Score } & NRS $0-2$ & & & & \\
\hline & NRS 3 & & & & \\
\hline & NRS $4-5$ & $C(, 014)$ & & & $C(, 009)$ \\
\hline \multirow[t]{2}{*}{ BMI unter oder gleich bis über $24 \mathrm{~kg} / \mathrm{m}^{2}$} & $<24 \mathrm{~kg} / \mathrm{m}^{2}$ & $\begin{array}{l}C(, 002) \\
D(, 002) \\
D(, 016)\end{array}$ & & & \\
\hline & $\geq 24 \mathrm{~kg} / \mathrm{m} 2$ & & $A(, 016)$ & $A(, 002)$ & $A(, 002)$ \\
\hline \multirow[t]{3}{*}{ Anzahl Pflegediagnosen 3 Kategorien } & tief $0-2$ & & & & \\
\hline & normal $3-4$ & & & & \\
\hline & hoch $>4$ & & & & \\
\hline \multirow[t]{3}{*}{ Pflegediagnose Ernährung } & Nicht vorhanden & & & & \\
\hline & vorhanden & & & & \\
\hline & Nicht nötig & &,$^{a}$ &,$^{a}$ & \\
\hline \multirow[t]{3}{*}{ Anzahl medizinischer Diagnosen } & $<6$ & & & & \\
\hline & $6-8$ & & & & \\
\hline & $>8$ & & & & \\
\hline
\end{tabular}

Anmerkungen: Die Ergebnisse beruhen auf zweiseitigen Tests. Für jedes signifikante Paar wird die Erläuterung der Kategorie mit den kleineren Spaltenanteilen in der Kategorie mit den größeren Spaltenanteilen angezeigt, Signifikanzniveau für Großbuchstaben (A, B, C): 0,05, ${ }^{a}$ Diese Kategorie wird nicht in die Vergleiche einbezogen, da ihr Spaltenanteil gleich Null oder 1 ist. ${ }^{b}$ Mit Hilfe der Bonferroni-Korrektur werden die Tests an alle paarweisen Vergleiche innerhalb einer Zeile der jeweils innersten Untertabelle angepasst. 
Eingeschlossene Patient_innen mit BMI $<24 \mathrm{~kg} / \mathrm{m}^{2}$ und NRS 4-5 zeigten auf, dass auch Patient_innen mit einer Gefahr für Mangelernährung während des Krankenhausaufenthaltes einen guten Bedarfsdeckungsgrad erreichen können. Dies bestärkt die Sinnhaftigkeit und Notwendigkeit des interprofessionellen Ernährungsmanagements ab Spitaleintritt.

\section{Stärken und Limitationen}

Eine Stärke dieser Studie besteht im Einschluss von Daten älterer hospitalisierter Patient_innen inklusive jener mit veränderter Kognition wie Demenz oder Delir aus verschiedenen Behandlungsbereichen. Zwei Drittel der Stichprobe waren Frauen, was der demografischen Verteilung in dieser Alterskategorie entspricht (Bundesamt für Statistik, 2020b).

Als mögliche Limitation ist zu nennen, dass die Datenqualität von Essprotokollen, NRS-2002 und weiteren ernährungsbezogenen Daten eventuellen Ungenauigkeiten unterliegen könnte, zumal nicht-randomisierte Routinedaten erhoben wurden.

Des Weiteren konnten aufgrund der begrenzten zeitlichen Ressourcen und kürzerer Liegedauer anstelle der geplanten je 110 Patient_innen insgesamt 45 Fälle pro Behandlungsbereich eingeschlossen werden. Dadurch lag die Anzahl Patient_innen je Gruppe bei 27 bis 39 Fällen. Die Bestimmung von statistisch signifikanten Unterschieden ist folglich limitiert, eine Verallgemeinerbarkeit der Ergebnisse verunmöglicht. Hingegen kann die Heterogenität der Stichprobe (Fallauswahl von drei Behandlungsbereichen mit unterschiedlichsten medizinischen Diagnosen) als Chance für eine Setting- und altersbezogene Repräsentativität gesehen werden.

Patient_innen mit Liegedauer $<5$ Tagen wurden ausgeschlossen, was als weitere Limitation angesehen werden könnte. Da jedoch bei Patient_innen mit längeren Krankenhausaufenthalten das Risiko für Mangelernährung besonders hoch ist (Woodward et al., 2020) lohnt es sich, diese Patient_innen und Zusammenhänge mit deren Ernährungssituation zu analysieren.

\section{Implikationen für die Pflegepraxis}

Die Kalorien- und Proteinbedarfsdeckungsgrade von älteren Patient_innen könnten auch in den Behandlungsbereichen Medizin und Chirurgie durch ein ernährungsförderndes interprofessionelles Behandlungskonzept mit systematischer Einschätzung der Gefahr einer Mangelernährung verbessert werden. Pflegefachpersonen sollten im interprofessionellen Team dahingehend geschult werden, dass ein hoher BMI die Gefahr einer Mangelernährung erhöht und dass Ernährungszustand sowie Nahrungszufuhr täglich überprüft werden müssen. Interprofessionelle, interdisziplinäre Praxisentwicklungs- und Qualitätssicherungsprojekte müssen dieses Vorgehen und die Haltung, dass Ernährung ein relevanter Teil der Pflege und Therapie ist, in allen Behandlungsbereichen berücksichtigen.

Weitere Forschung zum Phänomen „Gefahr einer Mangelernährung“ in mehreren Akutspitälern wird benötigt, um ein ernährungsförderndes interprofessionelles Behandlungskonzept zu entwickeln und zu implementieren.

\section{Schlussfolgerungen}

Protein- und Kalorienbedarfsdeckungsgrade hängen mit dem Behandlungsbereich und folglich mit dessen Teamkultur und Behandlungskonzept zusammen. Aufgrund der Eigenschaften der Gruppe „Gut-Ernährter“ wird klar, dass interprofessionelle, interdisziplinäre Behandlungsteams mit strukturierten Rapporten, dem systematischen Miteinbezug der Ernährungstherapeut_innen und einer für alle sichtbaren Dokumentation der Gefahr von Mangelernährung älterer Patient_innen entgegenwirken können.

\section{Elektronische Supplemente (ESM)}

Die elektronischen Supplemente sind mit der Online-Version dieses Artikels verfügbar unter https://doi.org/10. 1024/1012-5302/a000844.

ESM1. Abbildungen S1-S4 und Tabellen S1-S16.

ESM2. Dendrogramm.

\section{Literatur}

Barker, L. A., Gout, B. S. \& Crowe, T. C. (2011). Hospital malnutrition: prevalence, identification and impact on patients and the healthcare system. International Journal of Environmental research and public health, 8(2), 514-527.

Bauer, S., Halfens, R. J. G. \& Lohrmann, C. (2017). Changes in nutritional status in nursing home residents and associated factors in nutritional status decline: a secondary data analysis. Journal of Advanced Nursing, 73(10), 2420 - 2429.

Beck, S., Geser, C. \& Grob, D. (2012). Multidimensionales geriatrisches Assessment als klinischer Zugang zum multimorbiden Patienten im Spital. Praxis, 101(1), 1627 - 1632.

Bundesamt für Statistik. (2020a). Medizinisches Kodierungshandbuch. Der offizielle Leitfaden der Kodierrichtlinien in der Schweiz. Vorabversion 2021. Neuchâtel: Federal Statistical Office.

Bundesamt für Statistik. (2020b). Alter, Zivilstand, Staatsangehörigkeit. Verfügbar unter https://www.bfs.admin.ch/bfs/de/home/ statistiken/bevoelkerung/stand-entwicklung/alter-zivilstandstaatsangehoerigkeit.html [15.06.2021].

Döring, N. \& Bortz, J. (2016). Forschungsmethoden und Evaluation in den Sozial- und Humanwissenschaften (5ed.). Heidelberg: Springer Verlag.

Gomes, F., Baumgartner, A., Bounoure, L., Bally, M., Deutz, N. E., Greenwald, J. L. et al. (2019). Association of Nutritional Support With Clinical Outcomes Among Medical Inpatients Who Are Malnourished or at Nutritional Risk: An Updated Systematic Review and Meta-analysis. JAMA Network Open, 2(11), e1915138.

Hager, I. (2019). Teil 1: Grundbegriffe und wichtigste Testverfahren (1. Auflage). Österreich: myMorawa.

Harris, J. A. \& Benedict, F. G. (1919). A biometric study of basal metabolism in man. Proceedings of the National Academy of Sciences of the United States of America., 4(12), 4. 
Hill, E., Subchaturas, W. \& Kloiber, O. (2013). Declaration of Helsinki - Ethical Principles for Medical Research involving Human Subjects. Verfügbar unter https://www.wma.net/policies-post/ wma-declaration-of-helsinki-ethical-principles-for-medicalresearch-involving-human-subjects/ [09.12.2017].

IBM. (2020). SPSS Statistic Version 26. USA: IBM.

ICH Harmonised Guideline (2016). Integrated Addendum to ICH E6(R1): Guideline for Good Clinical Practice E6(R2). Verfügbar unter http://www.ich.org/fileadmin/Public_Web_Site/ICH_Pro ducts/Guidelines/Efficacy/E6/E6_R2__Step_4_2016_1109. pdf [09.12.2017].

Kantonale Ethikkomission Zürich. (2017). Vorgehen bei Gesuchseinreichung. Verfügbar unter https://kek.zh.ch/internet/gesund heitsdirektion/kek/de/home.html [09.09.2017].

kofam. (2017). Koordinationsstelle Forschung am Menschen (kofam). Verfügbar unter www.kofam.ch [09.09.2017].

Kondrup, J., Allison,S. P.,Elia, M., Vellas, B., Plauth, M. (2003). ESPEN Guidelines for Nutrition Screening 2002. Clinical Nutrition, 22(4), 415-421.

Malone, M. L., Capezuti, E. A., \& Palmer, R. M. (2014). Acute Care for Elders. A Model for Interdisciplinary Care (1 ed.). Springer Science and Business Media, https://doi.org/10.1007/978-1-4939-1025-0

Mayer, H. (2019). Pflegeforschung anwenden. Elemente und Basiswissen für das Studium und Weiterbildung. [Applying nursing research. Elements and basic knowledge for studying and further education] (5. Auflage). Wien: facultas.wuv Universitätsverlag.

Müller, F. S., Meyer, O. W., Chocano-Bedoya, P., Schietzel, S., Gagesch, M. L., Freystaetter, G. et al. (2017). Impaired nutritional status in geriatric trauma patients. European journal of clinical nutrition, 71(5), 602-606.

Ottrey, E., Porter, J., Huggins, C. E. \& Palermo, C. (2018). "Meal realities" - An ethnographic exploration of hospital mealtime environment and practice. Journal of Advanced Nursing, 74(3), 603-613.

Polit, D. F., \& Beck, C.T. (2020). Nursing research. Generating and assessing evidence for nursing practice. (11 ed.). Philadelphia: Wolters Kluwer.

Rosenberger, C., Rechsteiner, M., Dietsche, R. \& Breidert, M. (2019). Energy and protein intake in 330 geriatric orthopedic patients: Are the current nutrition guidelines applicable? Clinical nutrition: official journal of the European Society of Parenteral and Enteral Nutrition, 29, 86-91.

Schwarz, J. \& Bruderer Enzler, H. (2018). Methodenberatung. Verfügbar unter https://www.methodenberatung.uzh.ch/de/daten analyse_spss/interdependenz/gruppierung/cluster.html [13.08.2018].

Stadtspital Waid und Triemli, Zürich. (2020). Universitäre Klinik für Akutgeriatrie. Verfügbar unter https://www.stadt-zuerich.ch/ waid/de/index/fachgebiete/akutgeriatrie.html [13.12.2020].

Stein, P., \& Vollnhals, S. (2011). Grundlagen clusteranalytischer Verfahren. [Unterrichtsmaterial - Working paper]. Duisburg.

Stöcklin, M. (2010). Statistik 1. Eine Einführung mit SPSS 16. Basel: [Vortragsmanuskript].

Tappenden, K. A., Quatrara, B., Parkhurst, M. L., Malone, A. M., Fanjiang, G. \& Ziegler, T.R. (2013). Critical role of nutrition in improving quality of care: an interdisciplinary call to action to address adult hospital malnutrition. JPEN Journal of Parenteral and enteral Nutrition, 37(4), $482-497$.

Vanderwee, K., Clays, E., Bocquaert, I., Verhaeghe, S., Lardennois, M., Gobert, M. et al. (2011). Malnutrition and nutritional care practices in hospital wards for older people. Journal of Advanced Nursing, 67(4), $736-746$.

Volkert, D., Beck, A. M., Cederholm, T., Cruz-Jentoft, A., Goisser, S., Hooper, L. et al. (2019). ESPEN guideline on clinical nutrition and hydration in geriatrics. Clinical Nutrition, 38(1), $10-47$.

von Elm, E., Altman, D.G., Egger, M., Pocock, S.J., Gotzsche, P. C. \& Vandenbroucke, J. P. (2008). The Strengthening the Reporting of Observational Studies in Epidemiology (STROBE) statement: guidelines for reporting observational studies. Journal of Clinlical Epidemiology, 61(4), $344-349$.

Woodward, T., Josephson, C., Ross, L., Hill, J., Hosking, B., Naumann, F. et al. (2020). A retrospective study of the incidence and characteristics of long-stay adult inpatients with hospital-ac- quired malnutrition across five Australian public hospitals. European journal of clinical nutrition, 74, 1668-1676.

Zanini, M., Bagnasco, A., Aleo, G., Timmins, F. \& Sasso, L. (2017). Returning to the sacred - the importance of careful attention to patients' nutritional needs in hospital settings. Journal of Advanced Nursing, 73(3), $523-526$.

\section{Historie}

Manuskripteingang: 01.02.2021

Manuskript angenommen: 27.09.2021

Onlineveröffentlichung: 25.10.2021

\section{Autorenschaft}

Substanzieller Beitrag zu Konzeption oder Design der Arbeit: $\mathrm{SB}, \mathrm{HM}, \mathrm{MB}, \mathrm{MMS}$

Substanzieller Beitrag zur Erfassung, Analyse oder Interpretation der Daten: SB, HM, KB, MB, MD, MMS

Manuskripterstellung: SB, HM, MMS

Einschlägige kritische Überarbeitung des Manuskripts: $\mathrm{HM}, \mathrm{KB}, \mathrm{MB}, \mathrm{MD}, \mathrm{MMS}$

Genehmigung der letzten Version des Manuskripts:

$\mathrm{SB}, \mathrm{HM}, \mathrm{KB}, \mathrm{MB}, \mathrm{MD}, \mathrm{MMS}$

Übernahme der Verantwortung für das gesamte Manuskript: $\mathrm{SB}, \mathrm{HM}, \mathrm{KB}, \mathrm{MB}, \mathrm{MD}, \mathrm{MMS}$

\section{Danksagung}

Vielen Dank an A. Heilbronner und P. Witschi, Leiter der Pflegeabteilung, für den unterstützenden Arbeitsvertrag für diese Studie. An Prof. Dr. M. Müller Staub und Univ.-Prof. Dr. H. Mayer geht ein grosses Dankeschön für die Betreuung und das Feedback während des Forschungs- und Schreibprozesses. Den Mitgliedern des Soundingboards gebührt grosse Anerkennung für die regelmässig stattfindenden Diskussionen des Forschungsplanes und der Ergebnisse sowie für die Begutachtung dieses Manuskripts. Die Mitglieder des Soundingboards sind: A. Bernhard, M. Breidert, MD PD; M. Dietrich, MD PD; S. Frei, K. Hopbach, M. Nardi, MD; und M. Rechsteiner, BScN. Zudem möchte ich mich ebenfalls erkenntlich zeigen bei I. Hager, welche mich bei der quantitativen Datenanalyse auf unkomplizierte Weise beraten hat.

Vielen Dank an die Pflegeteams und die Ernährungsberatung für die Datenerhebung: Teamleitungen sind Annette Augst, Nadine Klein, Sonja Perlick, Monica Rechsteiner.

\section{ORCID}

Silvia Brunner

(D) https://orcid.org/0000-0003-4460-882X

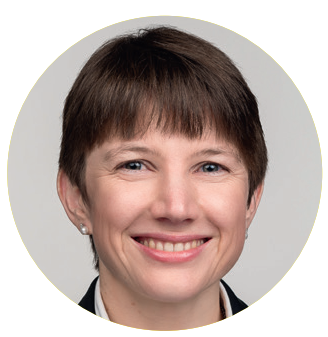

Silvia Brunner, MScN, PhDcand

Institut für Pflegewissenschaft

Universität Wien

Alser Strasse 23/12

1080 Wien

Österreich

a11734614@unet.univie.ac.at

\section{Was war die größte Herausforderung bei Ihrer Studie?}

Bei hoher Arbeitslast das Pflegefachpersonal zur Essprotokollführung zu motivieren.

Was wünschen Sie sich bezüglich der Thematik für die Zukunft? Durch genaue Einschätzung die Ernährung älterer Patient_innen im Krankenhaus zu verbessern.

\section{Was empfehlen Sie zum Weiterlesen /Vertiefen?}

Malone, Capezuti \& Palmer (2014). Acute Care for Elders. A Model for Interdisciplinary Care (1 ed.). Siehe Literatur. 